\title{
Repeat Detailed Second Trimester Ultrasound: Hawaii Experience
}

\author{
${ }^{1}$ Marguerite Lisa Bartholomew, ${ }^{2}$ Steve Shaha, ${ }^{3}$ Lynnae Millar, ${ }^{4}$ Ivica Zalud
}

${ }^{1}$ Assistant Professor, Division of Maternal Fetal Medicine, Department of Obstetrics, Gynecology and Women's Health, John A Burns School of Medicine, University of Hawaii, Honolulu, Hawaii, USA

${ }^{2}$ Associate Professor, Center for Public Policy and Administration, University of Utah, Salt Lake City, Utah, USA

${ }^{3}$ Professor and Chair, Division of Maternal Fetal Medicine, Department of Obstetrics, Gynecology and Women's Health, John A Burns School of Medicine, University of Hawaii, Honolulu, Hawaii, USA

${ }^{4}$ Professor and Chief, Division of Maternal Fetal Medicine and Obstetrics and Gynecology Imaging Division, Department of Obstetrics and Gynecology, John A Burns School of Medicine, University of Hawaii, Honolulu, Hawaii, USA

Correspondence: Marguerite Lisa Bartholomew, Department of Obstetrics, Gynecology and Women's Health, John A Burns School of Medicine, University of Hawaii, Kapi'olani Medical Center for Women and Children, Fetal Diagnostic Center, 1319 Punahou Street, Honolulu, Hawaii 96734, USA, Phone: (808)983-8559, Fax: (808)983-8989, e-mail: mbarthol@hawaii.edu

\begin{abstract}
This review is about clinical value of repeat second trimester ultrasound performed in a tertiary center in the detection of fetal anomalies. A retrospective record review was performed on all of the second trimester obstetrical ultrasounds (15 to 22 weeks) performed during a four month period. The ultrasound reports were reviewed to determine if repeat studies resulted in a change of diagnoses with respect to fetal anomalies. Ultrasound diagnoses were compared to newborn records to determine the accuracy of ultrasound diagnoses. 1,470 ultrasound examinations were performed on 1,344 patients. The rate of repeat examination was $8.41 \%$ after the exclusion of 13 patients with suboptimal views as the indication for the repeat ultrasound. $943(70.16 \%)$ newborn birth records were available for analysis. Fifty six $(6 \%)$ of the newborns were coded as having an anomaly at birth. $55 \%$ of these anomalies were detected by ultrasound, $36 \%$ were not detectable by ultrasound, and $9 \%$ were not detected by ultrasound. The overall fetal anomaly rate was $3.8 \%$. The overall detection rate was $\mathbf{8 6 . 1 \%}$. There were no diagnosis changes nor additional anomalies identified after repeat second trimester ultrasound.

Objectives

- Describe the role of comprehensive ultrasound in detection of fetal anomalies

- Present one highly specialized fetal diagnostic center experience

- Discuss the value of repeat ultrasound exams in order to increase anomaly detection rate
\end{abstract}

Keywords: Second trimester ultrasound, anatomy, anomalies, repeat ultrasound.

\section{ULTRASOUND AND CONGENITAL ANOMALIES}

Ultrasound diagnosis of congenital anomalies has been part of obstetric care for 30 years. The American Institute of Ultrasound in Medicine requires evaluation of fetal anatomy during a complete second trimester ultrasound, ${ }^{1}$ but gives no recommendation about when to offer follow-up ultrasound. In the hands of experienced sonographers and/or sonologists, the detection rates (sensitivity) for congenital abnormalities with routine second trimester ultrasound have been reported to be has high as $92 \% .{ }^{2}$ However, most studies using routine ultrasound indicate detection rates between 60 to $80 \%$ (range 16 to $92 \%, 7 / 14$ studies with rates greater than $70 \%) .{ }^{3}$ The ultrasound detection rates for patients at high-risk for congenital anomalies are the same or higher than routine ultrasound detection rates (range 11 to $99 \%$, $4 / 8$ studies with rates greater than $70 \%$ ). ${ }^{3}$ The specificity of ultrasound is high for both routine and high risk ultrasound is (91 to $100 \%)^{3}$

There is controversy concerning the impact of routine ultrasound on perinatal outcome despite the existence of several randomized trials. The largest randomized trial (RADIUS study) was performed in the United States. RADIUS study concluded that routine ultrasound did not improve perinatal morbidity and mortality, but did improve the detection rate of fetal anomalies. RADIUS demonstrated that routine ultrasound before 24 weeks detected $16.6 \%$ of anomalies compared to ultrasound when indicated (control group) which detected $4.9 \%$ of fetal anomalies ( $<<0.001) .{ }^{4}$ 
Conversely, the Helsinki randomized trial concluded that routine ultrasound decreased perinatal mortality by $49.2 \%{ }^{5}$ This difference was likely caused by the increased rate of elective abortion in the those with ultrasound detected anomalies.

A meta-analysis of four randomized clinical trials involving 15,935 pregnancies (not including RADIUS) showed that routine ultrasound not only decreased perinatal mortality, but also improved the detection rate of small for gestational age infants, multiple pregnancies, and severe malformations. ${ }^{6}$

The RADIUS study has been sharply criticized for a low overall detection rate (16.6\%) and inclusion of a large number of nontertiary care centers (63\%). Detection rates have been shown to be better in tertiary care centers or when ultrasounds are performed or reviewed by Maternal Fetal Medicine (MFM) specialists. ${ }^{7-9}$

It appears clear that routine ultrasound improves detection rates of anomalies, but what is the need for repeat ultrasound? Ultrasound diagnosis of fetal anomalies allows patients to make decisions about prenatal treatment, delivery in a tertiary care center, or pregnancy termination. Maternal habitus, fetal position, gestational age, and expertise all play a role in the ability to detect fetal abnormalities. Great variation also exists among ultrasound providers concerning recommendations for follow-up ultrasound. This review examines whether repeat second trimester ultrasound examinations performed in a tertiary center by maternal fetal medicine specialists improves the detection rate of congenital anomalies.

\section{HAWAII EXPERIENCE}

After approval by the institutional review board, a retrospective record review was performed. All singleton ultrasound examinations were done between 15 to 22 weeks at the Kapiolani Medical Center for Women and Children's Fetal Diagnostic Center in a four month period. All ultrasound examinations were performed by experienced physicians. The standard of care was to perform a detailed anatomy survey on all patients. Every ultrasound exam (initial and repeat) included documentation of number of fetuses, biometry, amniotic fluid, cardiac activity, fetal brain including lateral ventricles, cavum septi pellucidi, cerebellum and cisterna magna, nuchal occipital thickness, face, lips, spine, four chamber view, outflow tracts, chest, abdominal wall, cord insertions, cord vessel number, stomach, diaphragm, kidneys, bladder, bowel, gender, number and architecture of limbs, hands, feet, placenta, maternal uterus, cervix and ovaries. All studies were performed by the physician investigators in real time.

Initial ultrasound studies were differentiated from repeat studies to determine the repeat ultrasound rate. All repeat ultrasounds were included regardless of who recommended/ ordered the studies (MFM vs referring practitioner). The repeat ultrasound reports were reviewed to determine if repeat studies resulted in a change of impression or diagnosis with respect to fetal anomalies. Ultrasound diagnoses were compared to newborn records to determine accuracy of ultrasound diagnosis. Fetal growth, amniotic fluid, and placental abnormalities were not included. Risk factors for fetal anomalies were not evaluated outside of the indication for ultrasound and maternal age.

Congenital anomalies were initially identified with newborn birth record billing (CPT) codes. Ultrasound diagnoses were compared to birth record coded anomalies to determine matched diagnoses. Matched diagnoses (anomalies) were considered detected. Cases in which coded anomalies did not match ultrasound diagnoses (suspected missed or incorrect diagnoses) were reviewed by five physician investigators. The unmatched anomalies were categorized as detected by ultrasound, undetected by ultrasound, or undetectable by ultrasound. The overall rate of anomaly detection was calculated. Sample size calculations were performed. Seven hundred and twenty five initial ultrasounds and thirty eight repeat ultrasounds were needed to have an $80 \%$ power (alpha 0.05 ) to increase anomaly detection from 60 to $90 \%$, with a baseline congenital anomaly rate of $3 \%$.

There were 1,470 ultrasound examinations performed on 1,344 patients. During the study period $90.7 \%$ of patients had one ultrasound, $9.0 \%$ of patients had one repeat ultrasound, and $0.3 \%$ had two or more repeat ultrasounds. $7.8 \%$ of patients were 35 years or older at the first ultrasound exam and $18.8 \%$ of patients had a genetic amniocentesis during the study period. Table 1 provides the indications for the initial ultrasound exams. The most common indications for the initial ultrasound were advanced maternal age, uterine size discrepancy, abnormal maternal serum screen, and suspected fetal anomaly. The mean gestational age at the time of initial examination for cases without repeat examinations was 17.9 weeks $(n=1344, \mathrm{SD}=1.47)$. The mean gestational age at the time of initial examination for 
Repeat Detailed Second Trimester Ultrasound: Hawaii Experience

Table 1: Indications for initial antepartum ultrasound exam

\begin{tabular}{|c|c|c|}
\hline Diagnoses & $N$ & $\%$ \\
\hline Advanced maternal age & 320 & 23.81 \\
\hline Small for dates & 296 & 22.02 \\
\hline Large for dates & 172 & 12.80 \\
\hline Abnormal maternal serum screen & 135 & 10.04 \\
\hline Suspected fetal anomaly & 117 & 8.71 \\
\hline Unsure LMP & 111 & 8.26 \\
\hline Anatomy screen & 70 & 5.21 \\
\hline Family history of anomaly & 31 & 2.31 \\
\hline No specific indication & 14 & 1.04 \\
\hline Diabetes mellitus & 14 & 1.04 \\
\hline Known fetal anomaly & 9 & 0.67 \\
\hline Bleeding & 8 & 0.60 \\
\hline Medication exposure & 7 & 0.52 \\
\hline Fibroids & 6 & 0.45 \\
\hline Placenta previa & 6 & 0.45 \\
\hline SLE & 5 & 0.37 \\
\hline Chromosomal fetal anomaly & 3 & 0.22 \\
\hline Pelvic pain & 3 & 0.22 \\
\hline Undesired pregnancy & 3 & 0.22 \\
\hline Oligohydramnios & 2 & 0.15 \\
\hline Short cervex & 2 & 0.15 \\
\hline Adnexal mass & 2 & 0.15 \\
\hline Confirm cardiac activity & 1 & 0.07 \\
\hline Habitual aborter & 1 & 0.07 \\
\hline Isoimmunization & 1 & 0.07 \\
\hline Maternal Toxoplasmosis & 1 & 0.07 \\
\hline Parvovirus exposure & 1 & 0.07 \\
\hline Renal transplant & 1 & 0.07 \\
\hline Thallasemia trait & 1 & 0.07 \\
\hline Thyroid Disease & 1 & 0.07 \\
\hline Total & 1,344 & $100 \%$ \\
\hline
\end{tabular}

cases with repeat examinations was 17.4 weeks $(\mathrm{n}=126$, $\mathrm{SD}=1.30$ ). Table 2 provides the diagnoses recorded after the initial ultrasound exams. The rate of suboptimal views after the initial ultrasound was $17.5 \%$. Table 3 summarizes the 126 repeat ultrasounds by indication, yielding a repeat ultrasound rate of $9.38 \%$. Thirteen patients who underwent
Table 2: Diagnoses recorded after initial ultrasound exam

\begin{tabular}{|lcc|}
\hline Diagnoses & \multicolumn{1}{c}{ N } & $\%$ \\
\hline Normal & 965 & 71.8 \\
\hline Suboptimal views & 235 & 17.5 \\
\hline Unsure gestational age & 60 & 4.5 \\
\hline Multiple gestation & 27 & 2.0 \\
\hline Maternal diagnoses & 23 & 1.7 \\
\hline Gl/bowel/intestine anomaly & 9 & 0.7 \\
\hline Choroid plexus cyst & 6 & 0.4 \\
\hline Kidney anomaly (including pyelectasis) & 6 & 0.4 \\
\hline Fetal demise & 4 & 0.3 \\
\hline Single umbilical artery & 3 & 0.2 \\
\hline Hydrops fetalis & 1 & 0.1 \\
\hline Skeletal dysplasia & 1 & 0.1 \\
\hline Twin-twin transfusion syndrome & 1 & 0.1 \\
\hline Anencephaly & 1 & 0.1 \\
\hline Amnion/Chorion unfused & 1 & 0.1 \\
\hline Hydrocephalus & 1 & 0.1 \\
\hline Total & $\mathbf{1 4 4}$ & $\mathbf{1 0 0} \%$ \\
\hline
\end{tabular}

a repeat ultrasound for the indication of suboptimal views were then excluded from the analysis leaving 113 patients and a repeat rate of $8.41 \%$. The rate at which individual sonologists recommended a repeat ultrasound exam ranged from 3.7 to $11 \%$. $17.70 \%$ of repeat exams were ordered for the indication of suspected fetal anomaly. The mean gestational age at the time of repeat examination was 19.9 weeks $(\mathrm{n}=126, \mathrm{SD}=1.75)$. The mean time between initial and repeat ultrasound exams was $18.1( \pm 6.4)$ days. The rationale was to allow enough time for better visualization of desired anatomy (e.g. different fetal position) but on the other hand not to have too long interval between exams to allow for comparable evaluation. All anomalies were detected during the first ultrasound. There were no diagnosis changes as a result of a repeat ultrasound, and no additional anomalies were detected. Power analysis for statistical interpretation of 113 repeat ultrasounds indicated a Power of 0.9857 (alpha $=0.05$ ) for evaluating whether a repeat rate is greater than $0.01 \%$.

Nine hundred and forty three newborn birth records (70.16\%) were available for analysis. There were no stillbirths. The mean gestational age at birth was 38.1 weeks, SD $=2.11$. Fifty-six of the 946 newborns (6\%) were coded 
Table 3: Indications for repeat ultrasound exam

\begin{tabular}{|lcc|}
\hline Anomaly & $N$ & $\%$ \\
\hline Advanced maternal age & 22 & 17.19 \\
\hline Suspected fetal anomaly & 20 & 15.63 \\
\hline Abnormal maternal serum screen & 16 & 12.50 \\
\hline Small for dates & 16 & 12.50 \\
\hline Suboptimal views & 13 & 10.16 \\
\hline Known fetal anomaly & 11 & 8.83 \\
\hline Large for dates & 6 & 4.69 \\
\hline Polyhydramnios & 6 & 4.69 \\
\hline Rupture of membranes & 3 & 2.34 \\
\hline Bleeding & 2 & 1.56 \\
\hline Fibroids & 2 & 1.56 \\
\hline No specific indication & 2 & 1.56 \\
\hline Diabetes mellitus & 2 & 1.56 \\
\hline Abdominal pain & 1 & 0.78 \\
\hline Family history of anomaly & 1 & 0.78 \\
\hline Maternal ovarian cyst & 1 & 0.78 \\
\hline Medication exposure & 0.78 \\
\hline Oligohydramnios & 126 & 0.78 \\
\hline Total & $100 \%$ \\
\hline
\end{tabular}

as having an anomaly at birth. Thirty-one of the 56 newborns with birth anomalies (55\%) were detected by ultrasound. Twenty newborns were determined to have birth anomalies that were undetectable by ultrasound (20/56 or 36\%). Please, see Table 4 . The remaining 5 anomalies were classified as undetected. (5/56 or 9\%) (Figure 1). One newborn had two anomalies (small ASD and VSD) (Table 5). The overall fetal anomaly rate was 3.8\% (36 newborns with anomalies/ 943 live births). The overall detection rate was $86.1 \%$ (31 cases detected by ultrasound/36 newborns with detectable anomalies).

\section{LITERATURE OVERVIEW}

The baseline risk of a congenital anomaly is reported to be between 2 to $5 \%{ }^{10,11}$ Our findings appear commensurate with this rate. In addition, the $86.1 \%$ detection rate appears consistent with detection rates published by other maternalfetal medicine specialists. ${ }^{8}$ The five missed diagnoses were relatively minor except for the Tetralogy of Fallot. The cardiac septal defects were small, managed conservatively in the newborn period, and were unlikely to cause significant
Table 4: Newborns with undetected anomalies by antepartum ultrasound

\begin{tabular}{|l|l|}
\hline Anomaly & $N$ \\
\hline Tetralogy of Fallot & \\
\hline $\begin{array}{l}\text { Ventricular septal } \\
\text { defect (VSD } 3 \mathrm{~mm} \text { ) }\end{array}$ & 1 \\
\hline $\begin{array}{l}\text { Atrial septal } \\
\text { defect (ASD } 2 \mathrm{~mm})\end{array}$ & 1 \\
\hline Bifurcate thumb & 1 \\
\hline Equinovarus deformity & 1 \\
\hline Total & 1 \\
\hline
\end{tabular}

${ }^{*}$ A heart defect was suspected but not specifically identified on first exam. Patient did not return for recommended repeat exam.

Table 5: Newborns with undetectable anomalies by antepartum ultrasound

\begin{tabular}{|l|c|}
\hline Anomaly & N \\
\hline Coding error - no anomaly & 4 \\
\hline Birthmark & 4 \\
\hline Patent ductus arteriosus & 3 \\
\hline Ear tag & 2 \\
\hline Innocent heart murmur & 2 \\
\hline Hypospadias & 2 \\
\hline Hypotonicity & 2 \\
\hline Facial asymmetry & 1 \\
\hline Total & $\mathbf{2 0}$ \\
\hline
\end{tabular}

long-term health problems. The Tetralogy of Fallot would likely have been definitively diagnosed if the patient had returned for the recommended follow-up. The physician suspected a malformation, but could not be definitive as a result of maternal obesity.

Many fetal anomalies are dependent on ultrasound for diagnosis. Congenital heart anomalies are the most common fetal anomalies, ${ }^{12}$ and are not detectable by maternal serum screening or routine prenatal care. Fetal heart malformations are commonly missed by ultrasound. ${ }^{13,14}$ The combination of the four chamber view and the left ventricular outflow tract (5 chamber view) improves detection rates over that of the four chamber view alone. ${ }^{15}$ Cardiac anomalies were the most common anomaly that went undetected in our study population. Three out of five fetuses with missed diagnoses had cardiac anomalies. Despite this finding, two of the three infants with undetected congenital heart disease had small septal defects that were clinically insignificant. 


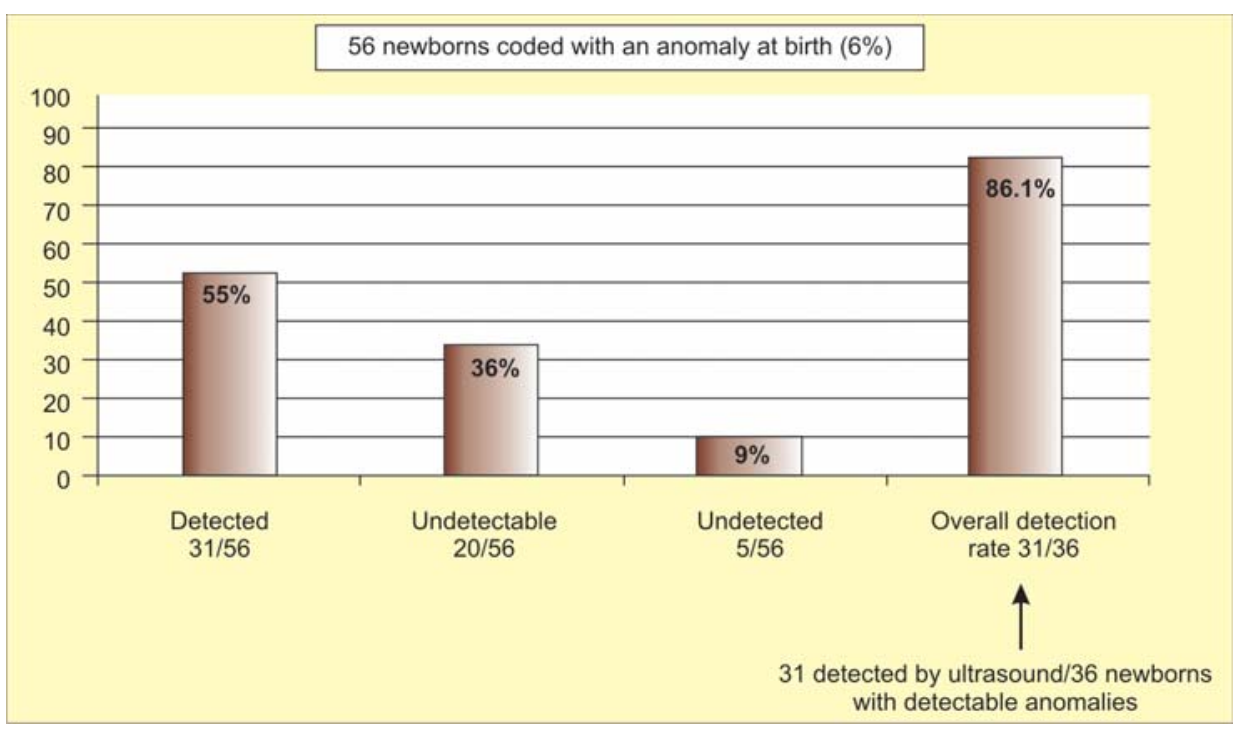

Figure 1: Results of anomaly detection by antepartum ultrasound

In clinical practice, repeat obstetrical ultrasound examinations are recommended for a variety of reasons. The majority (65\%) of repeat ultrasound indication in our study were related to fetal anatomy concerns (advanced maternal age, suspected fetal anomaly, abnormal maternal serum screen, and known fetal anomaly). Ten percent of the repeat examinations were ordered for suboptimal views and these were excluded from analysis. There is little in the literature addressing the potential benefit of routine repeat ultrasound for completion of fetal anatomy visualization. One abstract by Persutte et al. was identified. ${ }^{16}$ There were 7505 exams on 2683 patients performed in a tertiary care center (2.8 exams/patient). Sixty-six percent of initial exams were considered suboptimal for one or more organ systems. The investigators noted that when inadequate visualization occurred, an anomaly was seen in only $0.2 \%$ of patients. When adequate visualization occurred, a major fetal anomaly was later detected in only $1.1 \%$ of patients. The authors concluded that follow-up examination to complete an incomplete examination is neither clinically or cost-effective. The mean gestational age at the initial ultrasound was somewhat late (23 weeks) compared to 17 weeks in our study.

In recent study, Chasen evaluated the impact of indicated (restricted) vs routine use of "detailed" second trimester sonography. ${ }^{17}$ Records of singleton pregnancies undergoing evaluation from 2004 to 2008 were reviewed. A detailed examination [Current Procedural Terminology (CPT) code 76811] was routinely performed on all patients. Major structural abnormalities were categorized on the basis of whether the structure would be included in a "basic" examination (CPT code 76805). Major anomalies were identified in 218 patients, 75 of whom elected to undergo abortion. In 88 patients (40.4\%), the abnormal structure would not be included in a basic examination. Risk factors were not more prevalent in those with anomalies requiring a detailed examination for diagnosis or in those patients who chose to undergo abortion. The authors concluded that the restricting detailed evaluation to those with risk factors would have prevented detection of a substantial proportion of anomalies.

The strengths of the study include the detailed ultrasound reports on all subjects, the low rate of missed diagnoses, and the large overall sample size that met an $80 \%$ power for initial and repeat scans to increase anomaly detection from 60 to $90 \%$. We acknowledge the small number of repeat ultrasounds and lack of stratification by indication for repeat ultrasound. We did not have enough patients with suboptimal views after the first exam to determine if repeat studies are valuable in this group simply because views were suboptimal. Although our review did not directly address the question of suboptimal views, it provides some support that in experienced hands scanning in real time, the majority of anomalies can be detected during the initial second trimester ultrasound exam as early as 17 weeks and that a second exam would not increase significantly the detection of anomalies. Other limitations of the review include its retrospective nature and that risk factors for congenital 
anomalies were not evaluated outside of the indication noted on the ultrasound report. The retrospective design could be a strength because it reflects actual practice patterns which would not be possible in a prospective study designed to maximize repeat exams. We did not collect information on infants born outside our hospital nor did we track fetuses that were electively aborted or spontaneously lost prior to viability. Newborn diagnoses were dependent on coding. Despite the high-rate of matched diagnoses, the accuracy of the billing codes could come into question. Newborn follow-up was limited to the newborn period leaving later diagnoses unknown.

\section{CONCLUSION}

Our experience indicates that repeat detailed ultrasound in the second trimester did not change the diagnosis when the examinations were performed in a highly specialized tertiary center. The majority of the repeat exams were indicated and only $10 \%$ were performed for suboptimal views on the first exam. Large prospective studies with attention to stratification of risk factors, operator experience, suboptimal first exams, and follow-up on terminated or spontaneously lost fetuses are needed to more precisely define recommendations for repeat second trimester ultrasound.

\section{ACKNOWLEDGMENTS}

We would like to thank the following physicians participating in ultrasound exams performed at the Fetal Diagnostic Center, Kapiolani Medical Center for Women and Children, Honolulu, Hawaii: Greigh Hirata, MD, Roy Nakayama, MD and Jana Silva, MD.

\section{REFERENCES}

1. AIUM Practice Guideline for the Performance of Obstetric Ultrasound Examinations: http://www.aium.org/publications/ guidelines/obstetric.pdf Accessed January 28, 2010.

2. Smith NC, Hau C. A six years study of the antenatal detection of fetal abnormality in six Scottish health boards. Br J Obstet Gynaecol 1999;106:206-12.

3. Goncalves LF, Romero R, Maymon E, Pacora P, Bianco K, Jeanty P. Prenatal detection of anatomic congenital anomalies. In: Fleischer A, Manning FA, Jeanty P, Romero R (Eds). Sono- graphy in Obstetrics and Gynecology Principles and Practice, Sixth Edition. New York: McGraw Hill; 2001:341-73.

4. Ewigman BG, Crane JP, Frigoletto FD, Le Fevre ML, Bain RP, McNellis D. A randomized trial of prenatal ultrasound screening in a low risk population: Impact on perinatal outcome. N Engl J Med 1993;329:931-37.

5. Saari-Kemppainen A, Karjalainen O, Ylostalo P, Heinonem OP. Ultrasound screening and perinatal mortality: Controlled trial of systemic one stage screening in pregnancy. The Helsinki Ultrasound Trial, Lancet 1990;336:387-91.

6. Bucher HC, Schmidt JG. Does routine ultrasound scanning improve outcome in pregnancy? Meta-analysis of various outcome measures. Br Med J 1993; 307:13-17.

7. Crane JP, LeFevre ML, Winborn RC, Evans JK, Ewigman BG, Bain RP, Frigoletto FD, McNellis D. A randomized trial of prenatal ultrasonographic screening: Impact on the detection, management, and outcome of anomalous fetuses. Am J Obstet Gynecol 1994;171:392-99.

8. Oguyemi D, Buskye S. Prenatal diagnosis of fetal anomalies in a regional tertiary center: The role of a maternal fetal medicine unit-a review of 6,877 deliveries. J Matern Fetal Med 2000;9: 219-23.

9. VanDorsten JP, Hulsey TC, Newman RB, Menard MK. Fetal anomaly detection by second trimester ultrasonography in a tertiary center. Am J Obstet Gynecol 1998;178:742-49.

10. Marden PM, Smith DW, McDonald MJ. Congenital anomalies in the newborn infant, including minor variations: A study of 4,412 babies by surface examination for anomalies and buccal smear for sex chromatin. J Pediatr 1964;64:357-71.

11. Chung CS, Myrianthopoulos NC. Congenital anomalies: Mortality and morbidity, burden, and classification. Am J Med Genet 1987;27:505-23.

12. Hoffman JIE, Christianson R. Congenital heart disease in a cohort of 19,502 births with long-term follow-up. Am J Cardiol 1978;42:641-47.

13. Vergani P, Mariani S, Ghidini A, Schiavina R, Cavallone M, Locatelli A, Strobolt N, Cerruti P. Screening for congenital heart disease with the four-chamber view of the fetal heart. Am J Obstet Gynecol 1992;167:1000-003.

14. Cullen S, Sharland GK, Allan LD, Sullivan ID. Potential impact of population screening for prenatal diagnosis of congenital heart disease. Arch Dis Child 1992:67;775-78.

15. Bromely B, Estroff JA, Sanders SP, Parad R, Roberts D, Frigoletto FD, Benacerraf BR. Fetal echocardiography: Accuracy and limitations in a population at high and low risk for heart defects. Am J Obstet Gynecol 1992; 166:1473-81.

16. Persutte WH, Jones III OW, Hobbins JC. Routine follow-up sonography to evaluate fetal structure is unnecessary (abstract). Am J Obstet Gynecol 1998;178:S12.

17. Chasen ST, Kalish RB, Chervenak FA. Basic versus detailed sonography: What do we miss? J Ultrasound Med 2009; 28(8):1015-18. 